\title{
Clinical significance of expression of Hintl and potential epigenetic mechanism in gastric cancer
}

\author{
HUAYING HUANG ${ }^{1}$, XIAOWEI WEI ${ }^{1}$, XIANWEI $\mathrm{SU}^{2}$, FENGCHANG QIAO ${ }^{2}$, \\ ZHI XU ${ }^{1}$, DONGYING GU ${ }^{1}$, HONG FAN ${ }^{2}$ and JINFEI CHEN ${ }^{1}$ \\ ${ }^{1}$ Department of Oncology, The Affiliated Nanjing First Hospital, Nanjing Medical University, \\ Nanjing 210006; ${ }^{2}$ Key Laboratory of Developmental Genes and Human Diseases, \\ Ministry of Education, Southeast University, Nanjing 210009, P.R. China
}

Received December 22, 2010; Accepted February 24, 2011

DOI: 10.3892/ijo.2011.994

\begin{abstract}
HINT1, a member of the evolutionary highly conserved HIT protein superfamily, is ubiquitously expressed in diverse species including mammalian tissues. Accumulating evidence shows that HINT1 is a haploinsufficient tumor suppressor. In the present study, we explored possible correlations between the expression level of HINT1 and clinicopathological features in tissues from gastric cancer (GC) patients. Decreased expression of HINT1 detected by qPCR and Western blotting in tumor tissues was found in 58.82 and $39.2 \%$ of the patients, respectively. Significantly reduced expression of HINT1 was found in poorly differentiated tumor tissues $(\mathrm{p}=0.027)$. Environmental interference (either H.pylori or EBV infection) $(p=0.005)$ was associated with the expression of HINT1. Moreover, compared with the GC tissues, the level of Hint1 detected by qPCR was significantly higher in the adjacent non-cancerous tissues $(\mathrm{p}=0.03)$. We treated AGS cells, a GC cell line with low expression level of HINT1, with 5 and $10 \mu \mathrm{mol} / 1$ 5-Aza-dC for $72 \mathrm{~h}$ and found that HINT1 could be induced by 5 -Aza-dC in a dose-dependent manner. These results suggested that Hintl expression was lower in GC tissues and some etiological factors, such as $H$. pylori/EB infection or promoter hypermethylation may play a role in gastric tumorigenesis.
\end{abstract}

\section{Introduction}

Gastric cancer (GC), as the fourth most common cancer and the second cause of death worldwide, causes nearly 1000,000 new cases and over 850,000 deaths every year (1). The epidemiology of GC has obvious geographic and demographic variations, occurring mainly in Africa and Asia. The infection of $H$. pylori is a common event and is shown to induce aberrant methylation in gastric cancer (2). Epstein-Barr (EB) virus infection is

Correspondence to: Dr Jinfei Chen, Department of Oncology, The Affiliated Nanjing First Hospital, Nanjing Medical University, 68 Changle Road, Nanjing, Jiangsu Province, P.R. China E-mail: jinfeichen@sohu.com

Key words: Hint1, GC, H. pylori, EB virus, promoter methylation considered as another important carcinogenic factor in gastric carcinogenesis (3). The development of GC is a complicated and multistep process involving multiple genetic and epigenetic aberrations, which cause cell proliferation, differentiation and genetic instability.

The HINT1 protein, as a member of the evolutionary highly conserved HIT protein super family, is ubiquitously expressed in diverse species including mammalian tissues. The HIT protein superfamily can be classified into the Hint branch, the Fhit branch and the GalT branch (4). In previous studies, Hintl knockout mice had a marked increase in susceptibility to chemical carcinogen-induced gastric tumors (5), mammary tumors (6) and ovarian tumors (6). These studies in mice provided evidence that HINT1 may be a haploinsufficient tumor suppressor. HINT1 protein can interact with several proteins and has corresponding effects, which include proapoptosis by up-regulation of p53 and Bax and down-regulation of Bcl-2 in human colon cancer cell line SW480 and breast cancer cell line MCF7 (7); cell cycle regulation by up-regulation of cellular levels of p27 (8); inhibition of several transcription factors including $\beta$-catenin (9), MITF (10), USF2 (11) and activator protein-1 (AP-1) (12); cellular responses to ionizing radiation via interaction with the product of ATDC gene to repress fos transcription (13); modulating the $\mu$-opioid receptor signaling pathway through interaction with the $\mu$-opioid receptor and/or RGSZ1 $(14,15)$. It had been reported that Hint1 is transcriptionally silenced in SW480 and increased expression of Hintl inhibits growth of the cell (12). Similar effects have been seen in non-small cell lung cancer (NSCLC) cell lines H522 and H538 $(9,17)$.

A growing number of genetic and epigenetic alterations in tumor suppressor and tumor-related genes, such as $A P C$, CHFR, COX2, DAP-kinase, DCC, E-cadherin, GSTP1, HRK, LOX, hMLH1, MGMT, p14, p15, pl6, PTEN, RASSF1A, RUNX3, 14-3-3 sigma, THBS1, TIMP-3 and TSLC1 (18-43), have been found to be involved in gastric carcinogenesis. In addition, more and more genes have been identified with aberrant promoter hypermethylation, suggesting that promoter hypermethylation is an important molecular mechanism for gastric carcinogenesis. In a previous study, promoter hypermethylation of Hintl was found in hepatocellular carcinoma (HCC) tissues and normal liver tissues (16), and cell lines such 
as SW480 (12), Hep3B and HepG2 (44). However so far there is no report suggesting the relationship of Hintl and GC tissues or GC cell lines.

Based on the above findings in the present study, we investigated Hintl protein and mRNA expression in GC and paired adjacent non-tumor tissues and its clinical significance.

\section{Materials and methods}

Tissue samples and cell lines. The human gastric cancer (GC) cell lines AGS and BGC-823 were obtained from cell bank of Chinese Academy of Sciences. Both the cell lines were maintained in medium containing RPMI-1640 (Invitrogen) supplemented with $10 \%$ fetal bovine serum. Cells were incubated in a $100 \%$ moist incubator with $5 \% \mathrm{CO}_{2}$ at $37^{\circ} \mathrm{C}$. $\mathrm{GC}$ and adjacent non-cancerous tissue specimens diagnosed by pathologists were obtained from the Nanjing First Hospital affiliated Nanjing Medical University. Tumor differentiation, depth of invasion, and lymph node metastasis was judged by routine pathological diagnosis. The clinicopathological features are shown in Table I. The age range was 38-91 years. Appropriate consent was obtained from each patient.

Reverse-transcription (RT)-PCR. Total RNA was isolated by using TRIzol Reagent (Invitrogen, USA) and first-strand cDNA was prepared from total RNA with Oligo(dT) 18 primer and AMV reverse transcriptase (BioFlux, Japan) according to the manufacturer's instructions. Primers used in subsequent PCR: Hint1: 5'-GAGATGGCAGATGAGATT-3' (forward) and 5'-TTAACCAGGAGGCCAATG-3' (reverse). PCR products were electrophoresed on $2 \%$ agarose gel, stained with ethidium bromide, and visualized under UV illumination. The intensities of the specific bands were analyzed and quantified.

Detection of H. pylori and EB virus in GC tissues. The presence of $H$. pylori and EB virus infection was determined by PCR for $H$. pylori $16 \mathrm{~S}$ rDNA and the EBNA-1 region, respectively. The primers for $H$. pylor $i$ were as follows: 5'-GCCAATGGTAA ATTAGTT-3' (forward) and 5'-CTCCTTAATTGTTTTTAC-3' (reverse). The primers for EB virus were as follows: 5'-GTCAT CATCATCCGGGTCTC-3' (forward) and5'-TTCGGGTTGGA ACCTCCTTG-3' (reverse). The DNA templates obtained from the GC patients were extracted by using the Biospin Tissue Genomic DNA Extraction Kit (BioFlux, Japan). H. pylori infection was also determined by Giemsa-stained histological staining. Cases that showed positivity in any assay were regarded as $H$. pylori-positive or EB virus-positive.

Real-time RT-PCR. To test HINT1 expression in normal GC tissues, Western blotting and real-time RT-PCR was carried out on the tissues. Primers used in the RT-PCR: Hint1: 5'-TTCTTCCGAGCCTCTCCTCT-3' (foward) and 5'-GACGA TACCCACCTCAGCAG-3' (reverse); $\beta$-actin: 5'-AAAGACCT GTACGCCAACAC-3' (foward)and5'-GTCATACTCCTGCTT GCTGAT-3' (reverse). Examination of the mRNA expression of Hintl was carried out by using a SYBR-Green PCR kit (Takara, Japan) under the following cycling conditions: PCR reactions were performed in $20 \mu 1$ of total volume with $2 \mathrm{U}$ polymerase (Takara) and cDNA samples equivalent to $0.5 \mathrm{ng}$ of RNA In 7300HT Fast Real-Time PCR System (Applied
Table I. Correlation of HINT1 expression level with clinicopathological features in $51 \mathrm{GC}$ cases.

\begin{tabular}{lrrrr}
\hline Parameters & Cases & $\mathrm{T}<\mathrm{N}$ & $\mathrm{T} \geq \mathrm{N}$ & P-value \\
\hline Age & & & & 0.839 \\
$\leq 60$ & 17 & 7 & 10 & \\
$>60$ & 34 & 13 & 21 & \\
Gender & & & & 0.839 \\
$\quad$ Male & 34 & 13 & 21 & \\
Female & 17 & 7 & 10 & \\
Differentiation & & & & $0.027^{\mathrm{a}}$ \\
$\quad$ Poor & 25 & 14 & 11 & \\
$\quad$ Moderate/well & 24 & 6 & 18 & \\
Invasive degree & & & & 0.665 \\
$\quad$ Early stage & 6 & 3 & 3 & \\
Progression & 43 & 16 & 27 & \\
& & & & 0.903 \\
Lymph node metastasis & & & & \\
$\quad$ Yes & 33 & 13 & 20 & \\
$\quad$ No & 17 & 7 & 10 & \\
EB infection & & & & 0.101 \\
$\quad$ Yes & & & & \\
No & 22 & 11 & 11 & \\
$\quad$ HP infection & 29 & 8 & 21 & \\
$\quad$ Yes & & & & 0.304 \\
$\quad$ No & 21 & 10 & 11 & \\
\hline
\end{tabular}

${ }^{\mathrm{a}}$ Significant difference.

Biosystems, USA). As an endogenous standard, $\beta$-actin was quantified synchronously in order to normalize the cDNA input among samples. The relative level of expression of Hintl among the different tissues was then calculated referring to the amount of $\beta$-actin (ABI PRISM 7300 Detection System, USA). Each reactions was performed in duplicate and the mean of the two experiments was used as the relative quantification value. At the end of 42 amplification cycles, the reaction products were collected and electrophoresed on 3\% agarose gel, to confirm that non-specific products were not obtained during the process of amplification.

5-Azadc treatment of GC cell. 5-Azadeoxycytidine (5-Aza$\mathrm{dC}$ ) was purchased from Sigma (USA). The cell line AGS was cultured at $2 \times 10^{5}$ in dishes on the first day. After incubated for $24 \mathrm{~h}$ the cells were treated with solvent or with 5-Azadc (5 or $10 \mu \mathrm{M})$ for $72 \mathrm{~h}$, and then their medium was changed to fresh for the last $24 \mathrm{~h}$. At last, the cells were harvested for RNA or protein extraction. All the assays were done in triplicate.

Protein extraction and Western blot assays. Cells were cultured and treated with the various conditions as described above. 
A

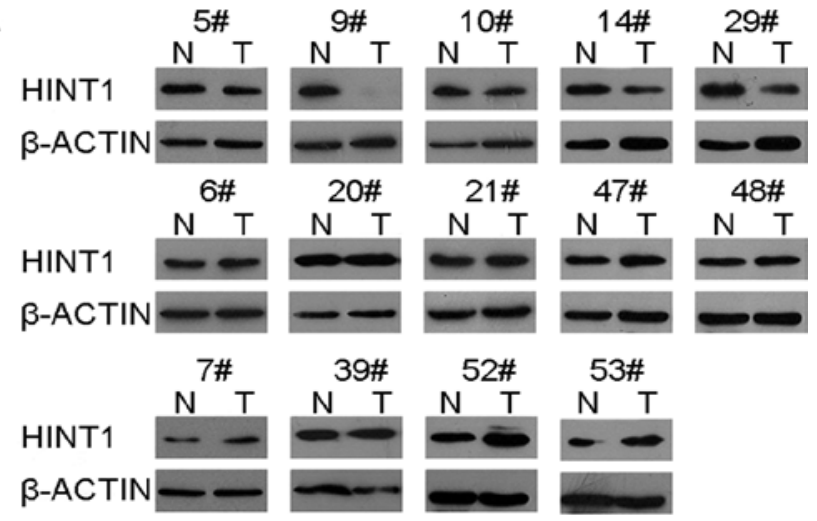

$B$

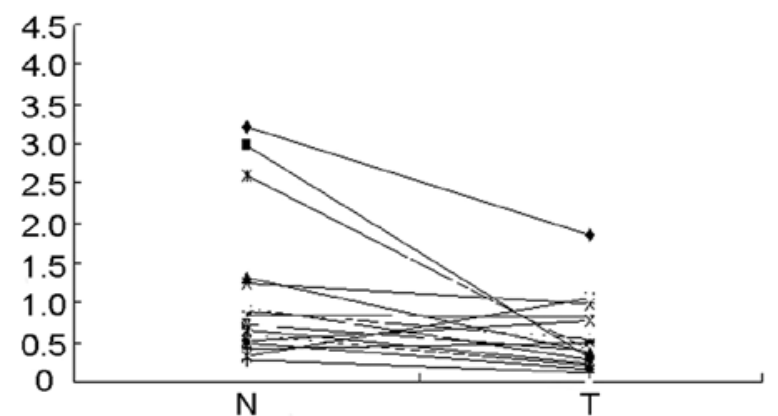

Figure 1. Expression of the Hint1 in GC and adjacent non-cancerous tissue samples. (A) Protein expression level was detected by Western blotting, 20 cases display $\mathrm{T}<\mathrm{N}$ and 27 cases display $\mathrm{T}=\mathrm{N}$ in the 51 pairs of $\mathrm{GC}$ and adjacent non-cancerous tissue samples. Representative examples are shown above. (B) In the RNA expression level detected by real-time RT-PCR, $58.82 \%$ of the cases display $\mathrm{T}<\mathrm{N}$ and $29.41 \%$ display $\mathrm{T}=\mathrm{N}$.

Protein extracts of tissues were then prepared as described. The proteins were separated by SDS-PAGE with $15 \%$ polyacrylamide gels and then blotted with the indicated antibody. The primary antibodies included anti-HINT1 antibody (ProteinTech Group, USA), anti- $\beta$-actin antibody (Sigma, USA). Anti-rabbit and anti-mouse IgG antibodies were used as the secondary antibodies, respectively. The intensities of specific protein bands were quantified with Gel Pro 3.2 (UVP, CLL, USA), corrected for the intensity of the respective $\beta$-actin band.

Immunohistochemistry (IHC) detection of HINT1 protein in paraffin-embedded sections. Tissue sections were obtained from Pathology Department of the Nanjing First Hospital affiliated Nanjing Medical University. IHC was used to detect the location and expression of HINT1. After deparaffinization in dimethylbenzene and rehydration in the gradient ethanol, the sections were heated in $10 \mathrm{mM}$ citric acid (pH 6.0) in a microwave oven for antigen retrieval. Staining was carried out according to the manufacturer's instruction: sections were incubated with the primary antibody (1:100 dilution) overnight at $4^{\circ} \mathrm{C}$, and then incubated with the secondary antibody $(1: 50$ dilution). Color development was carried by using $\mathrm{ABC}$ reagent and DAB. Slides were counterstained with hematoxylin. The following categories were used for scoring: percentage of positive staining, $<5 \%$ (0), $5-25 \%$ (1), $25-50 \%$ (2), $>50 \%$ (3) of cells, and intensity of staining, none (0), mild (1), moderate (2), strong (3). Combining percentage and intensity staining resulted in the following score $0-1$; negative (-); 2-6 positive (+).
Table II. Correlation of Hint1 mRNA expression level with clinicopathological features in $34 \mathrm{GC}$ cases.

\begin{tabular}{lrrrc}
\hline Parameters & Cases & $\mathrm{T}<\mathrm{N}$ & $\mathrm{T} \geq \mathrm{N}$ & P-value \\
\hline Age & & & & 0.29 \\
$\leq 60$ & 11 & 5 & 6 & \\
$>60$ & 21 & 15 & 6 & \\
& & & & 0.226 \\
Gender & & & & \\
$\quad$ Male & 23 & 16 & 7 & \\
Female & 10 & 4 & 6 & \\
& & & & \\
Differentiation & & & & 0.912 \\
$\quad$ Poor & 16 & 9 & 7 & \\
$\quad$ Moderate/well & 12 & 7 & 5 & \\
Invasive degree & & & & 0.492 \\
$\quad$ Early stage & 2 & 2 & 0 & \\
Progression & 24 & 13 & 11 & \\
& & & & 0.683 \\
Lymph node metastasis & & & & \\
$\quad$ Yes & 18 & 11 & 7 & \\
No & 8 & 4 & 4 & \\
EB infection & & & & 0.868 \\
$\quad$ Yes & & & 6 & \\
No & 14 & 8 & 6 & \\
HP infection & 20 & 12 & 8 & \\
$\quad$ Yes & & & & 0.226 \\
No & 17 & 8 & 9 & \\
\hline
\end{tabular}

Statistical analysis. SPSS 13.0 software for Windows (Microsoft, USA) was used for statistical analysis. Correlation of the expression of Hintl between GC and adjacent non-cancerous tissues were analyzed through $\chi^{2}$ test, differences were analyzed by the Fisher's exact test. Results were considered statistically significant at $\mathrm{p}<0.05$.

\section{Results}

Expression of Hintl in GCs and the corresponding normal gastric mucosae. The mRNA expression levels of the Hintl were detected by qPCR in 34 pairs of matched tumor and non-tumor tissues and decreased expression of Hintl was $58.82 \%$ in tumor tissues, while the protein expression of HINT1 was detected by Western blotting in 51 cases and decreased expression was $39.2 \%$ in tumor tissues comparing to the non-tumor tissues (Fig. 1). In addition, the mRNA expression level of Hintl in GC was significantly lower than that in non-tumor tissues ( $\mathrm{p}=0.030$ ) (Fig. 2).

Although we did not find any significant correlations between Hint1 mRNA expression levels and the clinicopathologic data (Table II), significantly decreased protein expression of HINT1 was observed in poorly differentiated tissues ( $p=0.027$, Table I). There was no obvious statistical 
A

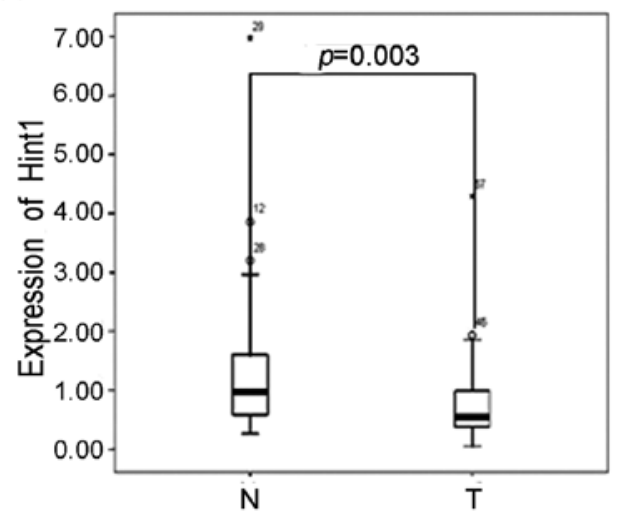

C

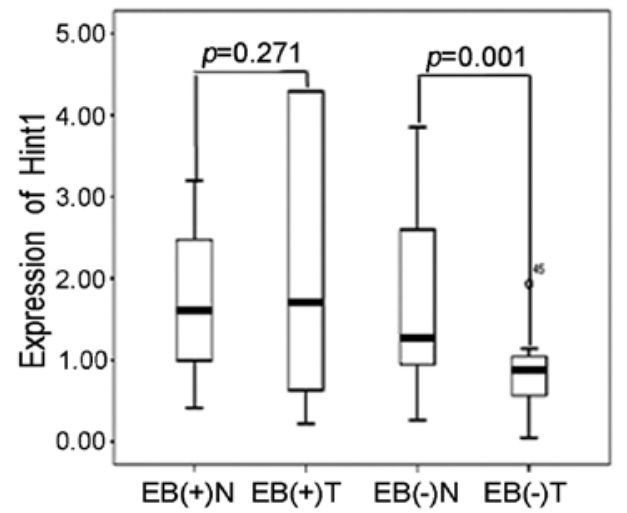

B

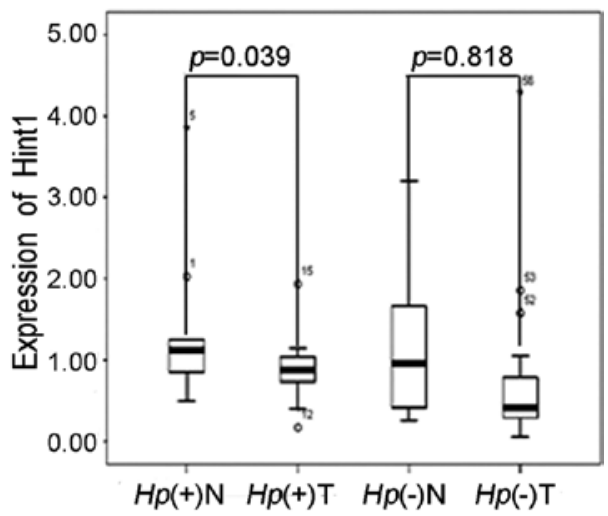

Figure 2. Relative Hint 1 mRNA expression levels were determined by qPCR from RNA preparation obtained from 34 pairs of GC and adjacent noncancerous tissue samples. The top and bottom horizontal lines of the boxes signify the 25th and 75th percentiles, respectively. The bold lines within the boxes indicate the median values, while the top and bottom horizontal bars show the maximum and minmum values, respectively. (A) When compared with the GC tissues the level of Hintl was significantly higher in the adjacent non-cancerous tissues ( $\mathrm{p}=0.03$ ). (B) In the H. pylori infection group, the expression level of Hintl was significantly higher in the adjacent noncancerous tissues $(\mathrm{p}=0.039)$. But there was no obvious statistical significance in the uninfection group. (C) However, in the EB-negative group, the expression level of Hintl was significantly higher in the adjacent noncancerous tissues $(\mathrm{p}=0.001)$. There was no obvious statistical significance in the positive group.

Table IV. The condition of $H$.pylori and EB infection detected by Western blot analysis. tumor invasive degree detected by Western blot analysis.

\begin{tabular}{lrrrc}
\hline & Cases & $\mathrm{T}<\mathrm{N}$ & $\mathrm{T} \geq \mathrm{N}$ & P-value \\
\hline EB infection & & & & \\
Yes & 22 & 11 & 11 & 1 \\
$\quad$ Early stage & 2 & 1 & 1 & \\
Progression & 18 & 9 & 9 & \\
No & 29 & 8 & 21 & 0.568 \\
Early stage & 4 & 2 & 2 & \\
Progression & 25 & 7 & 18 & \\
& & & & \\
HP infection & & & & \\
Yes & 21 & 10 & 11 & 1 \\
Early stage & 3 & 2 & 1 & \\
Progression & 17 & 8 & 9 & \\
No & 30 & 11 & 20 & 1 \\
$\quad$ Early stage & 3 & 1 & 2 & \\
Progression & 26 & 8 & 18 & \\
\hline
\end{tabular}

significance when we analyzed the relevance between $H$. pylori or EB infection and the result of Western blotting. We found obvious statistical significance when we combined the $H$. pylori and EB infection with the result of Western blotting (Table IV). Therefore, we could affirm that the environmental interference (either H. pylori or EB infection) was interrelated to lower expression of HINT1 in GC tissues.

\begin{tabular}{lrrrr}
\hline & Cases & $\mathrm{T}<\mathrm{N}$ & $\mathrm{T} \geq \mathrm{N}$ & P-value \\
\hline Hp and EB infection & & & & $0.007^{\mathrm{a}}$ \\
$\quad$ Both & 9 & 3 & 6 & \\
Either & 25 & 15 & 10 & \\
$\quad$ Neither & 17 & 2 & 15 & \\
Hp and EB infection & & & & $0.005^{\mathrm{a}}$ \\
$\quad(+)$ & 34 & 18 & 16 & \\
$(-)$ & 17 & 2 & 15 & \\
\hline
\end{tabular}

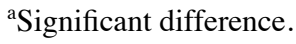

IHC was used to show the immunostaining of HINT1 in gastric mucosal cells. Representative examples of HINT1 expression in adjacent non-cancerous tissues showed expression of the HINT1 in both tumor cell nuclei and cytoplasm (Fig. 3B and D). GC tissue with moderate differentiation displayed expression of the HINT1 in both tumor cell nuclei and cytoplasm (Fig. 3A) while GC tissue with poor differentiation showed lack of expression in tumor cells (Fig. 3C). PBS substituted for the primary antibody of HINT1 and BGC-823 were used as blank and positive controls, respectively (Fig. 3E and F).

Expression of Hint 1 in GC cells. There is increasing evidence that Hintl is a novel tumor suppressor gene. In view of the 
A

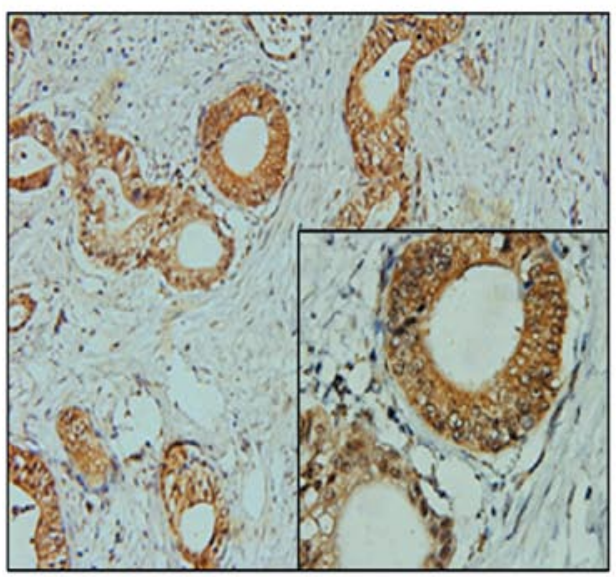

C

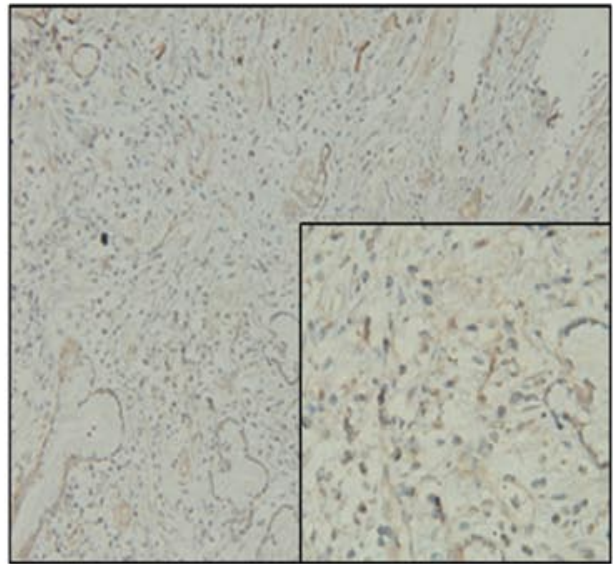

E

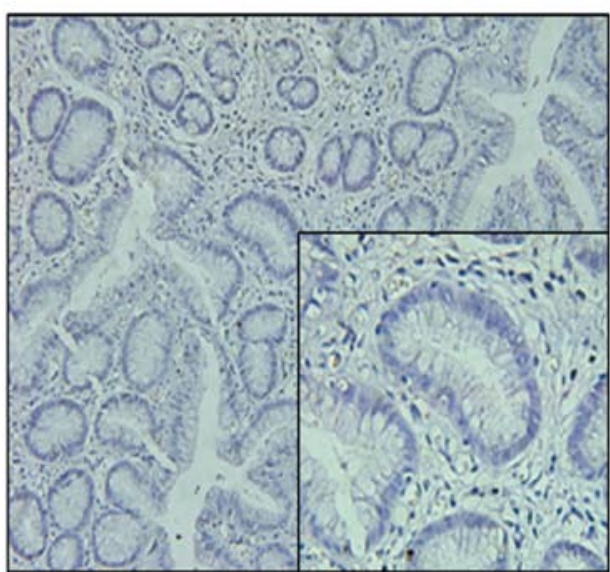

B

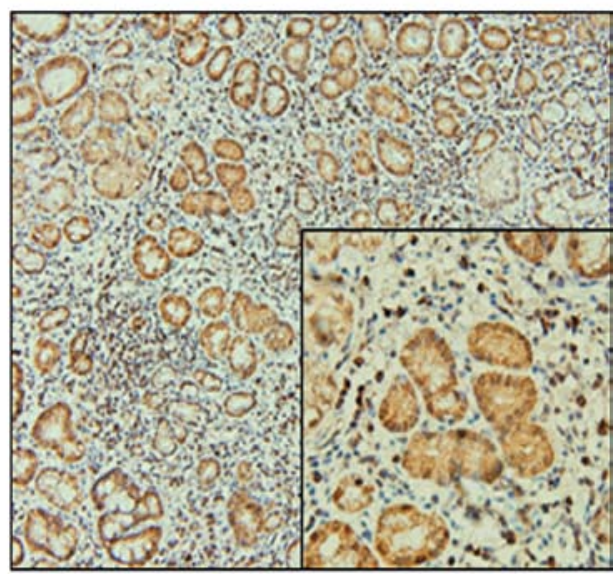

D

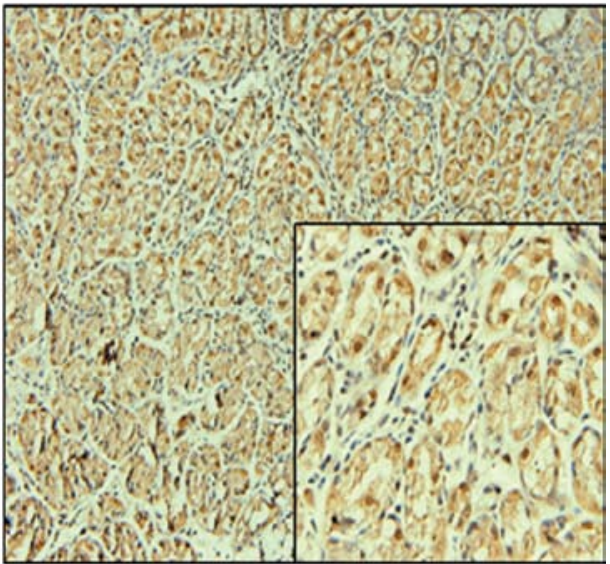

F

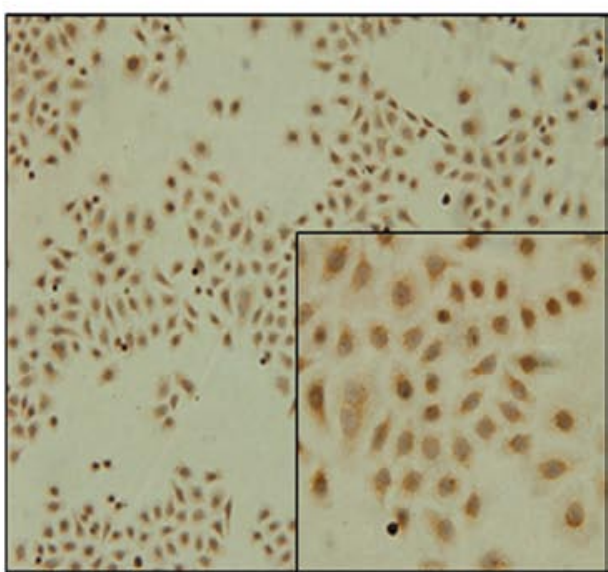

Figure 3. Immunohistochemical detection of HINT1 expression in GC cases. (A) GC tissue with moderate differentiation shows expression of the HINT1 protein in both tumor cell nuclei and cytoplasm, x200 and x400 (lower right corner). (B) Paired adjacent non-cancerous tissue of (A) shown expression of the HINT1 protein in both tumor cell nuclei and cytoplasm, x200 and x400 (lower right corner). (C) GC tissue with poor differentiation lacks expression in tumor cells, x200 and x400 (lower right corner). (D) Paired adjacent non-cancerous tissue of (C) shows expression of the HINT1 protein in both tumor cell nuclei and cytoplasm, x200 and x400 (lower right corner). (E) PBS substituted for the primary antibody of HINT1 and used as a blank control, x200 and x400 (lower right corner). (F) BGC-823 was used as a positive control, $\mathrm{x} 200$ and $\mathrm{x} 400$ (lower right corner).

low expression of Hint1 in GC cases, it was of interest to examine the level of Hintl in GC cell lines. Among the two GC cell lines, AGS displays lower level of Hintl than BGC-823. That is, the expression of Hintl was more significantly inhibited in GC cell line AGS. Similar results were obtained in repeat studies.
The level of Hintl ascends after AGS treatment by 5-Azadc. The expression of specific tumor suppressor genes is often inhibited in cancer cells via methylation of specific cytidine residues in the corresponding promoter regions of these genes and also via other modifications that alter chromatin structure. There was a previous study which suggested that the relatively 
A

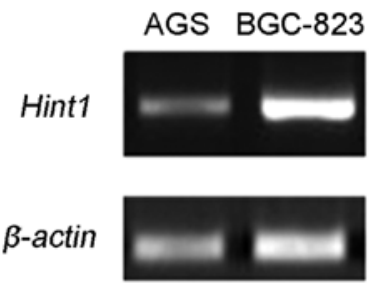

C

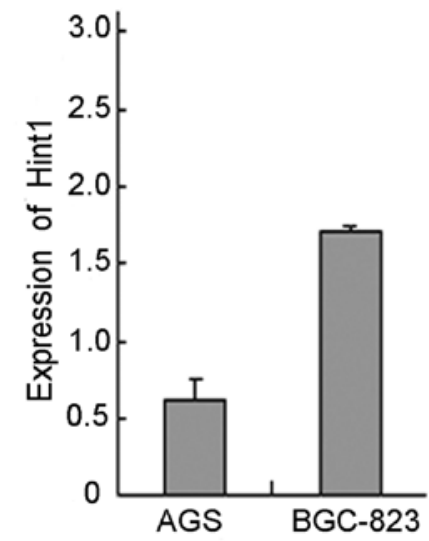

B

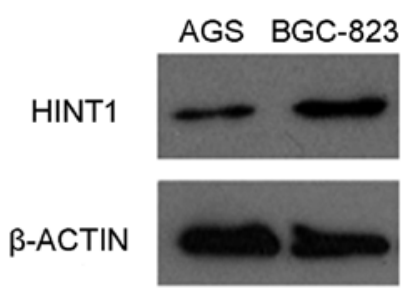

D

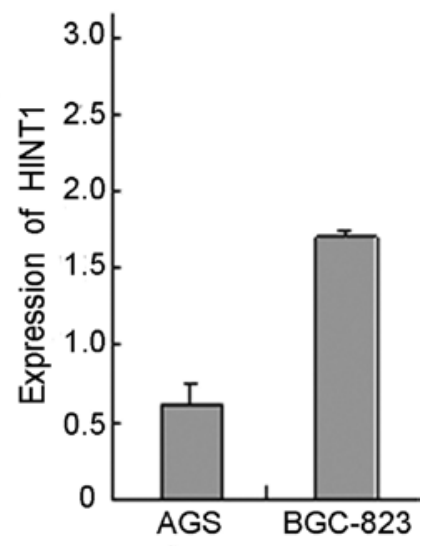

Figure 4. (A and C) The cells were cultured and then treated as described previously in Materials and methods. When it was detected by RT-PCR, the expression of Hintl in AGS was lower than that in BGC-823. (B and D) Similar result was confirmed by Western blotting. Expression ratios for HINT1 were calculated after normalization for $\beta$-actin. Assays were done in duplicate and the same results were obtained.

A

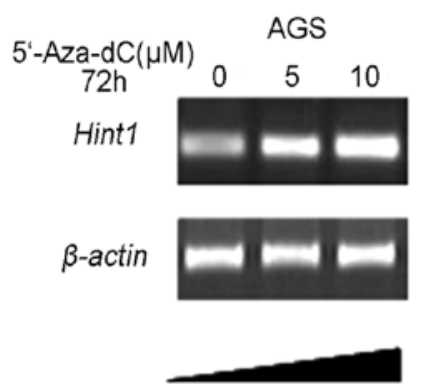

C

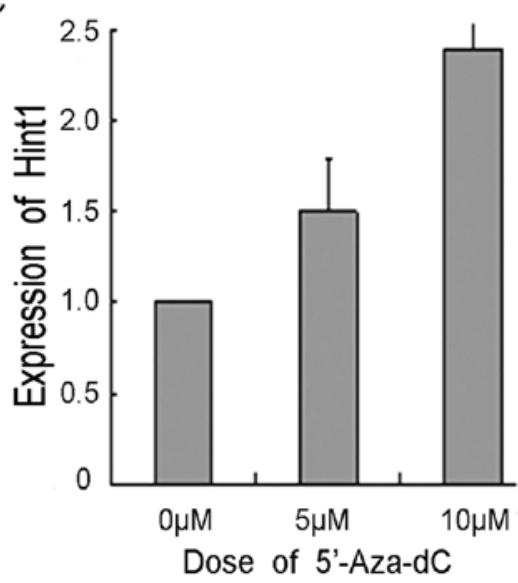

B

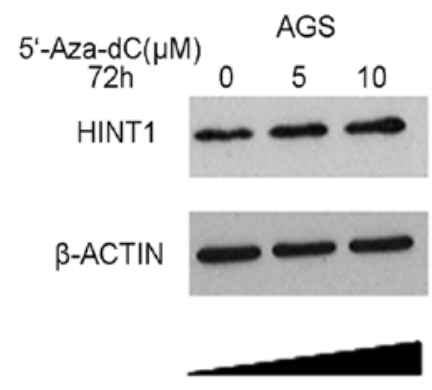

D

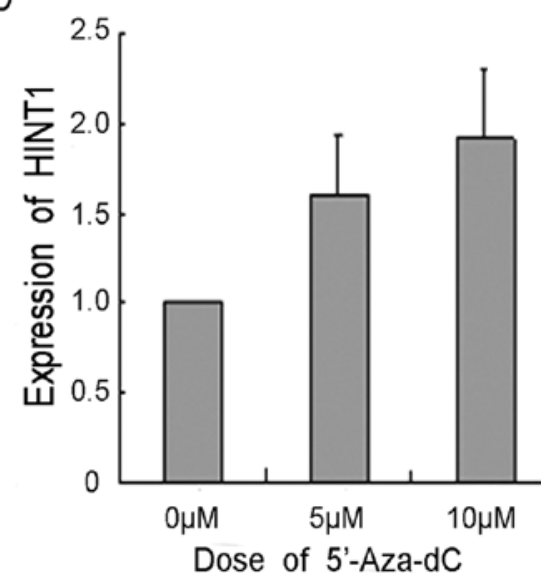

Figure 5. (A and C) The GC cell AGS was treated with 0, 5 and $10 \mu \mathrm{mol} / 1$ 5-Azadc for $72 \mathrm{~h}$. Total RNA was isolated and semiquantitative RT-PCR was done with specific Hint1 and $\beta$-actin (internal loading control) primers. PCR products were analyzed on $2 \%$ agarose gels and visualized under UV illumination. (B and D) Cells were treated with 5-Aza-dC as described above, and the same results were gained by Western blot analysis.

low levels of HINT1 in human HCC cell lines HepG2 and Hep3B (10), NSCLC cell line H522 (17) and colon cancer cell line SW480 (12) may be due, at lease in part, to promoter methylation. Therefore, we treated AGS with 5 and $10 \mu \mathrm{mol} / 1$ 
5-Aza-dC for $72 \mathrm{~h}$ and then examined by RT-PCR and Western blotting the levels of expression of HINT1 mRNA and protein, respectively. We found that the level of HINT1 could be induced by treatment with $5-\mathrm{Aza}-\mathrm{dC}$ and the elevation was dose-dependent. The same results were obtained in triplicate studies.

\section{Discussion}

Previous investigations with genetically engineered mice evidenced that Hintl is a novel haploinsufficient tumor suppressor gene (6). Its potential mechanism of tumor progression was characterized in human hepatocellular carcinoma (HCC) (16). However, the mechanism and clinical significance of HINT1 is unclear in other types of human cancer. In the present study, we examined HINT1 protein expression levels by Western blotting in tissues from GC patients and we found 20 cases showing $\mathrm{T}<\mathrm{N}, 70 \%$ (14 cases) displayed poor differentiation, but 29 cases showed $\mathrm{T} \geq \mathrm{N}$, only $37.93 \%$ (11 cases) displayed poor differentiation $(\mathrm{p}=0.027)$. This result suggested that comparing with moderately/well differentiated cases, the expression of HINT1 was decreased more in poorly differentiated GC tissues. Infection of $H$. pylori and EB are considered as important carcinogenic factors in gastric carcinogenesis (2,3). In the present study, we found at least in protein level either H. pylori or EB infection was associated with the reduced expression of HINT1 in GC tissues ( $\mathrm{p}=0.005)$. Moreover, in the $H$. pylori infection group, Hint 1 mRNA expression was significantly lower in the GC tissues than in the non-tumor tissues $(\mathrm{p}=0.039)$, while in the uninfection group, there was no significantly statistical difference. However, in the EB-negative group, the mRNA expression level of Hint 1 was significantly higher in the adjacent non-cancerous tissues ( $p=0.001$ ), whereas, there was no obvious statistical significance in the positive group. Therefore, we found that the protein level the expression of HINT1 was more likely repressed in GC tissues with poorly differentiated, H.pylori or EB infection. Furthermore, the mRNA expression of Hintl was suppressed more in $H$. pylori-positive and EB-negative GC tissues.

Previous studies indicate that HINT1 is recruited to ionizing radiation-induced foci (IRIF) and associates with $\gamma$-H2AX and ATM in response to ionizing radiation (IR)-induced or radiomimetic drug bleomycin-induced DNA damage responses, and plays an important role in modulating the appropriate responses to DNA damage in mammalian cells (45). In addition, HINT1-deficient cells exhibit resistance to IR-induced apoptosis and several types of chromosomal abnormalities $(5,45)$. Overexpression of HINT1 in the LM217 cell line increases the sensitivity of these cells to ionizing radiation (46). These findings clarify that HINT1 may play a role in radiation sensitivity at least by either ionizing radiation or radiomimetic drug such as bleomycin. As stated previously, we can suggest that patients with low or deficient expression of HINT1 may clinically appear resistant to radiotherapy or chemotherapy.

In the present study, we found HINT1 expression in AGS was lower than that in the other human GC cell line BGC-823. Previous investigations found decreased expression of Hint 1 in human HCC cell lines HepG2 and Hep3B is due to methylation of the promoter region of the Hint1 gene (44). Similar evidence was obtained in human NSCLC cell lines
(17) and colon cancer cell SW480 (12) based on studies using 5-Aza-dC. When we treated AGS with 5-Aza-dC we found that the level of HINT1 could also be induced and the elevation was dose-dependent. Thus, we suggest that decreased expression of Hint1 in human GC cell line AGS is, at least in part, due to methylation of the promoter region of the Hintl gene.

Based on the above findings, we investigated promoter hypermethylation of Hintl in several DNA samples from GC patients. In four cases with HINT1 underexpression, methylation bands were determined in GC tissues and unmethylation bands were determined in paired adjacent non-tumor tissues (data not shown). The result suggested that promoter methylation status may inversely correlate with the HINT1 expression in GC tissues.

Immunohistochemical (IHC) data showed that HINT1 was localized in both nuclei and cytoplasm in the majority of moderately differentiated GC tissues while it was underdetectable in poorly differentiated GC tissues. Besides, it was widely expressed in the paired adjacent non-cancerous tissues. These results were in line with the result from Western blotting. However, there were still 2 of 10 moderately diffe-rentiated and poorly differentiated GC tissues that showed inverse results (data not shown). Previous IHC study suggested that $83 \%$ of unmethylated HCC samples demonstrated positive nuclear and cytoplasmic staining, and 64\% of methylated HCCs showed loss of expression of HINT1, and in adjacent non-tumor samples $87 \%$ of unmethylated samples demonstrated positive nuclear and cytoplasmic staining and $53 \%$ of methylated samples showed loss of expression of HINT1 (16). Therefore, we can infer that in our study the 2 of 10 moderately differentiated GC tissues with lack of HINT1 expression detected by IHC may due to promoter methylation, and likewise the poorly differentiated GC tissues with positive HINT1 expression detected by IHC may due to unmethylation of the promoter.

In conclusion, this was the first study on detection of HINT1 expression in GC tissues. HINT1 was underexpressed in GC tissue in protein and mRNA levels. Its downregulation was associated with poorer tumor cell differentiation and viral infection such as by $H$. pylori/EB virus, suggesting patients with underexpressed HINT1 may have biologically aggressive tumor and poor prognosis. Underexpression of HINT1 in GC may also be due to promoter methylation and plays a role in gastric tumorigenesis.

\section{Acknowledgements}

This work was supported by Nanjing Medical Key Scientific Foundation, (no.: ZKX08012) and the Open Project Program of Key Laboratory of Developmental Genes and Human Diseases, Ministry of Education, Southeast University (no.: 200903). We are most grateful for the experimental platform provided by the Key Laboratory.

\section{References}

1. Japanese Gastric Cancer Association: Japanese classification of gastric carcinoma - 2nd English edition. Gastric Cancer 1: 10-24, 1998.

2. Nakajima T, Yamashita S, Maekita T, Niwa T, Nakazawa K and Ushijima T: The presence of a methylation fingerprint of Helicobacter pylori infection in human gastric mucosae. Int J Cancer 124: 905-910, 2009. 
3. Uozaki $\mathrm{H}$ and Fukayama M: Epstein-Barr virus and gastric carcinoma - viral carcinogenesis through epigenetic mechanisms. Int J Clin Exp Pathol 1: 198-216, 2008.

4. Brenner C: Hint, Fhit, and GalT: function, structure, evolution, and mechanism of three branches of the histidine triad superfamily of nucleotide hydrolases and transferases. Biochemistry 41 : 9003-9014, 2002.

5. Su T, Suzui M, Wang L, Lin CS, Xing WQ and Weinstein IB: Deletion of histidine triad nucleotide-binding protein 1/PKCinteracting protein in mice enhances cell growth and carcinogenesis. Proc Natl Acad Sci USA 100: 7824-7829, 2003.

6. Li H, Zhang Y, Su T, Santella RM and Weinstein IB: Hint1 is a haploinsufficient tumor suppressor in mice. Oncogene 25 713-721, 2006

7. Weiske $J$ and Huber $O$ : The histidine triad protein Hint1 triggers apoptosis independent of its enzymatic activity. J Biol Chem 281: 27356-27366, 2006.

8. Cen B, Li H and Weinstein IB: Histidine triad nucleotide-binding protein 1 up-regulates cellular levels of $\mathrm{p}^{27^{\mathrm{KIP} 1}}$ by targeting $\mathrm{Scf}^{\mathrm{SKP} 2}$ ubiquitin ligase and Src. J Biol Chem 284: 5265-5276, 2009.

9. Weiske $J$ and Huber $O$ : The histidine triad protein 1 interacts with Pontin and Reptin and inhibits TCF- $\beta$-catenin-mediated transcription. J Cell Sci 118: 3117-3129, 2005.

10. Razin E, Zhang ZC, Nechushtan H, Frenkel S, Lee YN Arudchandran R and Rivera J: Suppression of microphthalmia transcriptional activity by its association with protein kinase Cinteracting protein 1 in mast cells. J Biol Chem 274: 34272-34276, 1999.

11. Lee YN and Razin E: Nonconventional involvement of LysRS in the molecular mechanism of USF2 transcriptional activity in FceRI-activated mast cells. Mol Cell Biol 25: 8904-8912, 2005.

12. Wang L, Zhang Y, Li H, Xu Z, Santella RM and Weinstein IB: Hint 1 inhibits growth and activator protein-1 activity in human colon cancer cells. Cancer Res 67: 4700-4708, 2007.

13. Brzoska PM, Chen H, Zhu Y, et al: The product of the ataxiatelangiectasia group D complementing gene, ATDC, interacts with a protein kinase $C$ substrate and inhibitor. Proc Natl Acad Sci USA 92: 7824-7828, 1995.

14. Ajit SK, Ramineni S, Edris W, Hunt RA, Hum WT, Hepler JR and Young KH: RGSZ1 interacts with protein kinase C interacting protein PKCI-1 and modulates mu opioid receptor signaling. Cell Signal 19: 723-730, 2007.

15. Guang W, Wang H, Su T, Weinstein IB and Wang JB: A novel $\mu$-opioid receptor interactive protein, in receptor desensitization, phosphorylation, and morphine-induced analgesia. Mol Pharmacol 66: 1285-1292, 2004.

16. Zhang YJ, Li H, Wu HC, et al: Silencing of Hint1, a novel tumor suppressor gene, by promoter hypermethylation in hepatocellular carcinoma. Cancer Lett 275: 277-284, 2009.

17. Yuan BZ, Jefferson AM, Popescu NC and Reynolds SH: Aberrant gene expression in human non small cell lung carcinoma cells exposed to demethylating agent 5-aza-2'-deoxycytidine. Neoplasia 6: 412-419, 2004

18. Tamura G, Yin J, Wang S, et al: E-cadherin gene promoter hypermethylation in primarv human gastric carcinomas. J Natl Cancer Inst 92: 569-573, 2000.

19. Fleisher AS, Esteller M and Wang S: Hypemethylation of the $h M L H 1$ gene promoter in human gastric cancers with microsatellite instability. Cancer Res 59: 1090-1095, 1999.

20. Fleisher AS, Esteller M, Tamura G, et al: Hypermethylation of the $h M L H 1$ gene promoter is associated with microsatellite instability in early human gastric neoplasia. Oncogene 20 329-335, 2001

21. Suzuki H, Itoh F, Toyota M, Kikuchi T, Kakiuchi H, Hinoda Y and Imai K: Distinct methylation pattern and microsatellite instability in sporadic gastric cancer. Int J Cancer 83: 309-313, 1999.

22. Toyota M, Ahuja N, Suzuki H, et al: Aberrant methylation in gastric cancer associated with the $\mathrm{CpG}$ island methylator phenotype. Cancer Res 59: 5438-5442, 1999.

23. Endoh Y, Tamura G, Ajioka Y, Watanabe H and Motoyama T: Frequent hypermethylation of the $h M L H 1$ gene promoter in differentiated-type tumors of the stomach with the gastric foveolar phenotype. Am J Pathol 157: 717-722, 2000.

24. Tamura G, Sato K, Akiyama S, et al: Molecular characterization of undifferentiated-type gastric carcinoma. Lab Invest 81 : 593-598, 2001.

25. Tsuchiya T, Tamura G, Sato K, et al: Distinct methylation patterns of two $A P C$ gene promoters in normal and cancerous gastric epithelia. Oncogene 19: 3642-3646, 2000.
26. Byun DS, Lee MG, Chae KS, Ryu BG and Chi SG: Frequent epigenetic inactivation of RASSFIA by aberrant promoter hypermethylation in human gastric adenocarcinoma. Cancer Res 61: 7034-7038, 2001.

27. Leung WK, Yu J, Ng EK, et al: Concurrent hypermethylation of multiple tumor-related genes in gastric carcinoma and adjacent normal tissues. Cancer 91: 2294-2301, 2001.

28. Kang GH, Shim YH, Jung HY, Kim WH, Ro JY and Rhyu MG: $\mathrm{CpG}$ island methylation in premalignant stages of gastric carcinoma. Cancer Res 61: 2847-2851, 2001

29. Kang GH, Lee S, Kim WH, Lee HW, Kim JC, Rhyu MG and Ro JY: Epstein-Barr virus-positive gastric carcinoma demonstrates frequent aberrant methylation of multiple genes and constitutes $\mathrm{CpG}$ island methylator phenotype-positive gastric carcinoma. Am J Pathol 160: 787-794, 2002.

30. Sato K, Tamura G, Tsuchiya T, Endoh Y, Usuba O, Kimura W and Motoyama T: Frequent loss of expression without sequence mutations in the $D C C$ gene in gastric cancer. Br J Cancer 85 199-203, 2001

31. Sato K, Tamura G, Tsuchiya T, et al: Analysis of genetic and epigenetic alterations of the PTEN gene in gastric cancer. Virchows Arch 440: 160-165, 2002.

32. Honda T, Tamura G, Waki T, et al: Hypermethylation of TSLC1 gene promoter in gastric cancer. Jpn J Cancer Res 93: 857-860, 2002 .

33. Lee TL, Leung WK, Chan MW, et al: Detection of gene promoter hypermethylation in the tumor and serum of patients with gastric carcinoma. Clin Cancer Res 8: 1761-1766, 2002.

34. Li QL, Ito K and Sakakura C: Causal relationship between the loss of RUNX3 expression and gastric cancer. Cell 109: 113-124, 2002.

35. Sakata K, Tamura G, Endoh Y, Ohmura K, Ogata S and Motoyama T: Hypermethylation of $h M L H 1$ promoter in solitary and multiple gastric cancers with microsatellite instability. $\mathrm{Br} J$ Cancer 86: 564-567, 2002.

36. Satoh A, Toyota M, Itoh F, et al: DNA methylation and histone deacetylation associated with silencing $D A P$-kinase gene expression in colorectal and gastric cancers. Br J Cancer 86: 1817-1823, 2002.

37. To KF, Leung WK, Lee TL, et al: Promoter hypermethylation of tumor-related genes in gastric intestinal metaplasia of patients with and without gastric cancer. Int J Cancer 102: 623-628, 2002.

38. Waki T, Tamura G, Tsuchiya T, Sato K, Nishizuka S and Motoyama T: Promoter methylation status of $E$-cadherin, $h M L H 1$ and pl6 genes in non-neoplastic gastric epithelia. Am J Pathol 161: 399-403, 2002.

39. Waki T, Tamura G, Sato M, Terashima M, Nishizuka S and Motoyama T: Promoter methylation status of DAP-kinase and $R U N X 3$ genes in neoplastic and non-neoplastic gastric epithelia. Cancer Sci 94: 360-364, 2003.

40. Nakase Y, Sakakura C and Miyagawa K: Frequent loss of RUNX3 gene expression in remnant stomach cancer and adjacent mucosa with special reference to topography. Br J Cancer 92: 562-569, 2005.

41. Honda T, Tamura G, Waki T, Kawata S, Nishizuka S and Motoyama T: Promoter hypermethylation of the Chfr gene in neoplastic and non-neoplastic gastric epithelia. Br J Cancer 90: 2013-2016, 2004

42. Obata T, Toyota M, Satoh A, et al: Identification of HRK as a target of epigenetic inactivation in colorectal and gastric cancer. Clin Cancer Res 9: 6410-6418, 2003.

43. Kaneda A, Wakazono K, Tsukamoto T, et al: Lysyl oxidase is a tumor suppressor gene inactivated by rnethvlation and loss of heterozvgosity in human gastric cancers. Cancer Res 64 6410-6415, 2004.

44. Wang L, Li H, Zhang Y, Santella RM and Weinstein IB: Hint1 inhibits $\beta$-catenin/TCF4, USF2 and NFKB activity in human hepatoma cells. Int J Cancer 124: 1526-1534, 2009.

45. Li H, Balajee AS, Su T, Cen B, Hei TK and Weinstein IB: The HINT1 tumor suppressor regulates both $\gamma$-H2AX and ATM in response to DNA damage. J Cell Biol 183: 253-265, 2008.

46. Choi EK, Rhee YH, Park HJ, Ahn SD, Shin KH and Park KK: Effect of protein kinase $\mathrm{C}$ inhibitor (PKCI) on radiation sensitivity and c-fos transcription. Int J Radiat Oncol Biol Phys 49: 397-405, 2001. 